\title{
Encoding specificity and unidirectional associates in cued recall
}

\author{
RONALD LEY \\ State University of New York, Albany, New York 12222
}

\begin{abstract}
The first of two experiments confirmed the earlier findings of Humphreys and Galbraith (1975), that under silent-study encoding instructions, words assessed as strong elicitors of target words are effective extralist retrieval cues, whereas words assessed as weak elicitors are not. In the second experiment, predictions from the encoding specificity principle were confirmed with respect to the effectiveness of forward and backward retrieval cues in recall. Contrary to the conclusions of Humphreys and Galbraith, forward and backward unidirectional associates were equally effective as retrieval cues, provided subjects engaged in associative encoding (generated associations to the target words) at storage.
\end{abstract}

Humphreys and Galbraith's (1975) criticism of research purporting to demonstrate the encoding specificity principle (specifically the findings of Thomson \& Tulving, 1970; Tulving \& Thomson, 1973) centers on the encoding procedure used by Thomson and Tulving. These critics point out that: "In situations where evidence for the encoding specificity principle has been obtained, subjects studied pairs of words and expected to be able to use one member of the pair as a cue for the other member" (Humphreys \& Galbraith, 1975 , p. 709). In this procedure, the subject is presented visually with a target word accompanied by a cue word with instructions that the cue should be studied as a possible aid in recalling the target. Humphreys and Galbraith maintain that the way in which a target word is encoded may be influenced by the presence of the cue word (i.e., paired associates consisting of cue and target), with the expectation that the cue will be used as an aid in recall of the target. Thus, a target word presented in the absence of a cue word (as in single-trial free recall) may lead to a different encoding of the target word than if the cue word were present (as in the Thomson \& Tulving and Tulving \& Thomson studies).

In the research reported by Humphreys and Galbraith (1975), an attempt was made to test predictions from the encoding specificity principle by means of an encoding procedure wherein the target and cue words were not presented as pairs; that is, an encoding procedure typically used in single-trial free recall. Since a test of predictions from the encoding specificity principle requires specification of the cue present at

This research was supported in part by a Grant-in-Aid and Faculty Research Fellowship from the State University of New York Research Foundation. Requests for reprints should be sent to Ronald Ley, State University of New York at Albany, 1400 Washington Avenue, Albany, New York 12222. storage of the target, Humphreys and Galbraith selected unidirectional words from Keppel and Strand's (1970) list, that is, 20 words (A words) that were assessed as strong elicitors of their respective primary associates (B words). The average strength of the association from $\mathrm{A}$ to $\mathrm{B}$ was $38.8 \%$, whereas the $\mathrm{B}$ words (the associates of the $A$ words) were assessed as weak elicitors of the A words (the average strength of the association from B to A was $2.5 \%$ ).

Although the subjects in Humphreys and Galbraith's (1975) study were instructed at storage simply to study silently, the implicit assumption was made that the subjects would engage in associative encoding. Thus, subjects who were required to study and recall the A words were expected to generate (covertly) corresponding $\mathrm{B}$ words at storage, since the B words were strong associates of the A words. If these B words were covertly elicited by the A words at storage, according to the encoding specificity principle, the $B$ words should serve as effective retrieval cues at recall. For the subjects who were required to study and recall the B words, it was expected that they would not covertly generate $\mathrm{A}$ words at storage, since the A words were weak associates of the $B$ words. If these A words were not covertly elicited by the B words at storage, according to the encoding specificity principle, the A words should not serve as effective retrieval cues at recall. It should be noted that these predictions are exactly the opposite of predictions from generationrecognition models (e.g., Bahrick, 1970). That is, since $B$ words are strong extralist associates of the A words, the A words should be effective retrieval cues, whereas, since the $A$ words are weak extralist associates of the $B$ words, the B words should be ineffective extralist retrieval cues.

Humphreys and Galbraith (1975) concluded that the findings of their Experiment 2 were contrary to the predictions from the encoding specificity principle 
and consistent with those of the generation-recognition model. The A words were found to be effective extralist retrieval cues for recall of the B words, and the B words were found to be ineffective retrieval cues for recall of the A words.

In view of the important theoretical implications of these findings, the present study was designed for the purpose of (1) testing the reliability of the findings of Humphreys and Galbraith (1975, Experiment 2) and (2) testing the crucial assumption underlying Humphreys and Galbraith's procedure, the assumption that subjects engage in covert associative encoding at storage when instructed to study silently. This assumption is crucial because any conclusions from these findings that might be relevant with respect to the encoding specificity principle demand that the subjects who were required to study the A words associatively encoded them by means of covertly generating $\mathrm{B}$ words as associates to each corresponding A word.

\section{EXPERIMENT 1}

The purpose of this experiment was to test the reliability of Humphreys and Galbraith's (1975) primary findings, namely, that A words (strong elicitors of $B$ word associates) are effective extralist retrieval cues for recall of B words, but that B words (weak elicitors of A words) are ineffective extralist retrieval cues for recall of A words. This test of reliability was performed by rieans of a replication of Humphreys and Galbraith's Experiment 2.

\footnotetext{
Method

Design. As with the Humphreys and Galbraith experiment, the design was a 2 by 2 factorial in which cued vs. free recall and A vs. B words were between-subjects variables. Half of the subjects were in the free recall condition and half were in the cued recall condition. Half of the subjects in each recall condition studied a list which consisted of 4 filler words and $20 \mathrm{~A}$ words (the same words used by Humphreys and Galbraith) and the other half studied a list which consisted of 4 filler words and $20 \mathrm{~B}$ words (the same words used by Humphreys and Galbraith). The cued recall subjects who studied A words were cued with the $B$ words. The filler words and their associates were selected from Ley and Tesiny's (1975) list of 382 words and paralogs, on the basis of the absence of any apparent semantic relationship between the filler words and their corresponding associates, with the $20 \mathrm{~A}$ words and $20 \mathrm{~B}$ words. The cued recall subjects who studied $B$ words were cued with the A words. The same filler words and the associates used with the A list were used with the B list.

Subjects. The subjects were 100 undergraduates at the State University of New York at Albany, randomly assigned in groups of 25 to each of the four treatment conditions: cued recall of A words, cued recall of B words, free recall of A words, and free recall of B words. The subjects were run in two group sessions, one of which involved the 50 subjects who were presented with the $A$ words, and the other of which involved the 50 subjects presented with the B words.

Materials and procedure. As in the Humphreys and Galbraith (1975) study, all of the subjects tested in the same session received the same study list (20 randomly ordered target words,
}

with two filler words at the beginning of the list and two at the end). Response booklets were randomly passed out so that half of the subjects received a booklet containing free recall instructions and half received a booklet containing cued recall instructions. The subjects were instructed that they would see a short slide presentation containing words which they should remember for a subsequent test. They were also informed that they would perform some simple arithmetic calculations for a short time during the interval between the slide presentation and the test. Subjects were instructed in both the free and cued recall conditions to write down as many of the presented words as they could remember. In addition, subjects in the cued recall group were asked to write the words next to the appropriate cue words or at the bottom of the page if they did not see an appropriate cue word. The words were presented visually (via a Kodak Carousel slide projector) at a 5 -sec rate $(4 \mathrm{sec}$ for exposure of slide and $1 \mathrm{sec}$ for changing slide), and the interval between presentation and the test was (approximately) $30 \mathrm{sec}$. During the $30-\mathrm{sec}$ retention interval, the subjects worked at the arithmetic task that was presented or " page of the response booklet.

\section{Results and Discussion}

The mean number of words recalled per subject for each of the four treatment combinations are given in Table 1 along with the corresponding means obtained by Humphreys and Galbraith (1975). A cursory glance at the data of this table indicates the high degree of agreement between the results of the present experiment and those of Humphreys and Galbraith. In all conditions except the cued recall of $B$ words, the differences between the means of the corresponding cells of the two experiments are less than one-half of a word. Under the cued recall of B words, Humphreys and Galbraith's subjects recalled 1.84 words more than the subjects of the present experiment.

In view of the high degree of uniformity between the two experiments, it comes as no surprise that the analysis of variance of the data of the present experiment also matches that of Humphreys and Galbraith; that is, the number of $B$ words recalled was significantly greater than the number of A words $[\mathrm{F}(1,96)=14.75$, $\mathrm{p}<.001, \quad \mathrm{MSe}=9.76]$. There was no significant difference between recall conditions $[F(1,96)=.80$, $\mathrm{p}>.05]$, but there was a significant interaction between recall condition and type of word being recalled $[F(1,96)=5.92, p<.025]$. The statistical analyses of the simple effects of the present experiment also corresponded with those of Humphreys and Galbraith; that is, for the A words the difference between free

Table 1

Mean Number of Words Recalled per Subject in Experiment 1 and in Humphreys and Galbraith (1975)

\begin{tabular}{|c|c|c|c|c|c|c|}
\hline \multirow{3}{*}{$\begin{array}{c}\text { Recall } \\
\text { Condition }\end{array}$} & \multicolumn{3}{|c|}{ Experiment 1} & \multicolumn{3}{|c|}{ Humphreys \& Galbraith } \\
\hline & \multicolumn{2}{|c|}{ Word Type } & \multirow[b]{2}{*}{ Total } & \multicolumn{2}{|c|}{ Word Type } & \multirow[b]{2}{*}{ Total } \\
\hline & $\mathbf{A}$ & B & & A & B & \\
\hline $\begin{array}{l}\text { Cued } \\
\text { Free }\end{array}$ & $\begin{array}{l}8.39 \\
9.37\end{array}$ & $\begin{array}{l}12.31 \\
10.23\end{array}$ & $\begin{array}{l}20.70 \\
19.60\end{array}$ & $\begin{array}{l}8.65 \\
9.85\end{array}$ & $\begin{array}{l}14.15 \\
10.65\end{array}$ & $\begin{array}{l}22.80 \\
20.50\end{array}$ \\
\hline Total & 17.76 & 22.54 & & 18.50 & 24.80 & \\
\hline
\end{tabular}


Table 2

Correlation Coefficients Between C - F Recall Scores and Associative Strength of Relationship Between A and B Words

\begin{tabular}{|c|c|c|c|c|c|}
\hline \multirow{2}{*}{$\begin{array}{l}\text { Direction of } \\
\text { Relationship }\end{array}$} & \multirow{2}{*}{$\begin{array}{l}\text { Word Type } \\
\text { Recalled }\end{array}$} & \multirow[b]{2}{*}{ Experiment 1} & \multirow[b]{2}{*}{ Experiment 2} & \multicolumn{2}{|c|}{$\begin{array}{c}\text { Humphreys \& Galbraith } \\
(1975)\end{array}$} \\
\hline & & & & Experiment 1 & Experiment 2 \\
\hline A to $B$ & A & .05 & -.28 & -.08 & $.56^{*}$ \\
\hline$A$ to $B$ & B & .36 & $.63^{*}$ & .40 & .36 \\
\hline $\mathrm{B}$ to $\mathrm{A}$ & $\mathbf{A}$ & .18 & .28 & .23 & $.46^{*}$ \\
\hline $\mathrm{B}$ to $\mathrm{A}$ & B & -.01 & .18 & -.10 & -.10 \\
\hline
\end{tabular}

${ }^{*} r=.44, p=.05, d f=18$

and cued recall was not significant $[\mathrm{t}(48)=-1.11$, $\mathrm{p}>05]$, while for the $\mathrm{B}$ words the difference between free and cued recall was significant $[\mathrm{t}(48)=2.36$, $\mathrm{p}<.02]$.

In addition to overall recall scores, Humphreys and Galbraith (1975) examined the cuing effect on an item-by-item basis: "The number of subjects recalling a particular item in the free recall condition was subtracted from the number recalling that item in the cued recall condition. This calculation, referred to as the $\mathrm{C}-\mathrm{F}$ score, was performed for each of the 20 A words and each of the 20 B words" (p. 704). In keeping with Humphreys and Galbraith's analysis, $\mathrm{C}-\mathrm{F}$ scores were computed and correlations were calculated between the $\mathrm{C}-\mathrm{F}$ scores and the strength of the associations from $\mathrm{A}$ to $\mathrm{B}$ and $\mathrm{B}$ to $\mathrm{A}$ for recall of both the $A$ and $B$ words. The correlation coefficients obtained in the present experiment and those obtained by Humphreys and Galbraith are given in Table 2. Although Humphreys and Galbraith found significant correlations between the strength of the $A$ to $B$ associations and the $C-F$ scores for the A words $[\mathrm{r}(18)=.56, \mathrm{p}<.05]$ and the strength of the $B$ to $A$ associations and the $\mathrm{C}-\mathrm{F}$ scores for the $\mathrm{A}$ words $[\mathrm{r}(18)=.46, \mathrm{p}<.05]$ in their Experiment 2, none of the correlations of the present experiment was significant. However, the pattern of correlations obtained in the present experiment was similar to that obtained in Humphreys and Galbraith's Experiment 1, an experiment identical to the present experiment and Humphreys and Galbraith's Experiment 2, except that a mixed list was used. (In Humphreys and Galbraith's Experiment 1, B words were recalled more frequently than A words and the difference between recall conditions was not significant, but the interaction between the recall condition and type of word recalled was not significant.)

The purpose of the present experiment was to test the reliability of the findings of Humphreys and Galbraith (1975, Experiment 2). Except for the two significant correlation coefficients reported by Humphreys and Galbraith, the results of the present experiment provide strong support in favor of the high degree of reliability in their findings.

\section{EXPERIMENT 2}

The purpose of this experiment was to test the assumption underlying Humphreys and Galbraith's
(1975) procedure; that is, the assumption that subjects engage in associative encoding (generate associations to target words) at storage when they are instructed to study silently. This assumption, which is questionable (Dean \& Ley, 1977; Locascio \& Ley, 1972; Ley, Dean, \& Locascio, Note 1), is crucial to their experimental strategy, since if subjects do not generate associations at the time of storage or if associations generated do not correspond to the primary associates obtained from the Keppel and Strand (1970) norms, then the encoding specificity principle.would not predict that the B words would be effective cues for the recall of the $A$ words.

In the present experiment, subjects were instructed to generate associations silently (covertly) to the target words during the time of their presentation at storage. By following this procedure, there could be greater assurance that the subjects were engaging in associative encoding, thus increasing the likelihood that the B words would occur as associates to the $A$ words at the time of storage. Although the B words are unidirectional primary associates of the A words, an examination of the Keppel and Strand (1970) norms indicates that most of the A words occur as secondary or lower order associates of the B words. Thus, subjects instructed to generate multiple associations are likely to generate A words as associates of B words even though the $B$ words are unidirectional primary associates of the A words.

The predictions of this experiment would be exactly the same as those of Experiment 1 and Humphreys and Galbraith's (1975) Experiment 2. That is, according to the encoding specificity hypothesis, B words should serve as effective retrieval cues for the recall of $\mathbf{A}$ words, whereas, according to generation-recognition models, Bwords should not serve as effective retrieval cues for recall of the A words.

\section{Method}

Design and procedure. The design and procedure was the same as in Experiment 1 except that all the subjects under all of the four treatment combinations were given associative encoding instructions; that is, subjects were instructed to encode the target words by means of generating associations silently (covertly) to each word during the entire duration of the presentation of all of the words of the list.

Subjects. The subjects were 100 undergraduates at the State University of New York at Albany, 25 in each of the four treatment conditions. As in Experiment 1, the subjects were run in group sessions separated on the basis of the presentation of $\mathrm{A}$ or $\mathrm{B}$ words. 
Table 3

Mean Number of Words Recalled per Subject Under Associative Encoding Instructions

\begin{tabular}{crrr}
\hline \multirow{2}{*}{$\begin{array}{c}c \\
\text { Recall }\end{array}$} & \multicolumn{2}{c}{ Word Type } & \\
\cline { 2 - 3 } Condition & \multicolumn{1}{c}{ A } & B & Total \\
\hline Cued & 12.39 & 12.76 & 25.15 \\
Free & 8.40 & 10.00 & 18.40 \\
Total & 20.79 & 22.76 & \\
\hline
\end{tabular}

\section{Results and Discussion}

The mean number of words recalled per subject for each of the four treatment combinations are given in Table 3. The singular datum of this table is the mean number of A words recalled under conditions in which $B$ words served as cues $(M=12.39)$. The comparison of this mean (12.39) with the mean number of $B$ words recalled under conditions in which $A$ words served as cues $(M=12.76)$ makes it clear that $B$ words are as effective extralist cues as are $A$ words when the target words are associatively encoded at storage. This finding is consistent with the prediction from the encoding specificity hypothesis, and is inconsistent with the prediction from a generation-recognition model. The fact that the number of $A$ words recalled was not less than the number of $B$ words confirms the earlier observation that: "Most of the A words occur as secondary or lower order associates of the B words. Thus, subjects instructed to generate multiple associations are likely to generate $\mathrm{A}$ words as associates of $\mathrm{B}$ words even though the $\mathrm{B}$ words are unidirectional primary associates of the A words."

An analysis of variance of the data of the present experiment showed a pattern exactly the opposite to that obtained in Experiment 1 and Humphreys and Galbraith's (1975) Experiment 2. That is, unlike the earlier experiments: (1) the number of $B$ words recalled $(M=22.76)$ was not significantly greater than the number of $A$ words recalled $(M=20.79)$ $[F(1,96)=2.03, p>.05, \mathrm{MSe}=11.33] ;(2)$ the number of words recalled under cued recall $(M=25.15)$ was greater than the number of words recalled under free recall $(M=18.40) \quad[F(1,96)=25.51, p<.001]$; and (3) the interaction between recall condition and type of word recalled was not significant $[F(1,96)=.79$, $\mathrm{p}>.05]$.

In order to make the analysis of the present experiment completely comparable with Experiment 1 of the present study and Experiment 2 of the Humphreys and Galbraith (1975) study, analysis of corresponding simple effects were performed. Unlike the earlier experiments, the difference between free and cued recall of $A$ words was significant $[\mathrm{t}(48)=4.20, \mathrm{p}<.01]$; but like the earlier experiments, the difference between free and cued recall of $B$ words was also significant $[\mathrm{t}(48)=2.90, \mathrm{p}<.02]$.

The differences between Experiments 1 and 2 suggest that subjects do not necessarily engage in associative encoding when instructed to study silently. This hypothesis is tenable even though free recall scores under associative encoding instructions (Experiment 2) were not significantly different from free recall scores under silent-study instructions (Experiment 1). In an experiment dealing with the effect of associative reaction time on free recall, Locascio and Ley (1972) found that, although total free recall scores under silentstudy instructions $(M=18.52)$ were equal to total free recall scores under two different associative encoding conditions [instructions to generate a single association to each target unit $(M=18.05)$ or instructions to generate multiple associations to each unit $(M=18.45)]$, an interaction between encoding instructions and type of unit to be recalled obtained. The analysis of this interaction showed that subjects instructed to study silently recalled significantly more short-latency units than long-latency units, whereas subjects instructed to encode associatively (single or multiple associations to target units) did not. These results illustrate the important point that equality of overall recall scores is not a sufficient condition for concluding that the same encoding process underlies different encoding instructions.

Furthermore, if subjects do not necessarily engage in associative encoding when instructed to study silently, the encoding specificity hypothesis does not apply. Therefore, Humphreys and Galbraith's (1975) study does not pit predictions from the encoding specificity hypothesis against those from generation-recognition models. Unlike the encoding specificity hypothesis, generation-recognition models do not require that associative encoding take place. The results of Humphreys and Galbraith's study and those of the present Experiment 1 can be neatly explained by generation-recognition models. It is clear that under unspecified encoding instructions (silent study), extralist words (A words) are effective cues for recall of their unidirectional primary associates (B words), but the opposite does not obtain. There are, however, explanations other than those from generation-recognition models to account for the superior recall of B words in Experiment 1 when $\mathbf{A}$ words were given as cues. A careful examination of the differences between the words of the two lists suggests two explanations.

One, some of the subjects in Experiment 1 may have used some of the cues to help them to guess correctly the words of their list. It is conceivable that some subjects engaged in a retrieval strategy wherein they wrote their first associations to some of the cues for those words they could not recall. Since the B words were primary associates of the $A$ words, the probability that this strategy would lead to a correct response (a word of List B) can be estimated on the basis of the average strength of the association from $A$ to $B$ (viz., $\mathrm{p}=.388$ ), and the probability that $\mathrm{B}$ words would lead to a correct response (a word of List A) would be the average strength of the association from $B$ to $A$ (viz., 
$p=.025)$. Given these probabilities. the expected total "recall" score for List A. independent of memory, would be $7.76(.388 \times 20)$, whereas the expected total "recall" score for List B, independent of memory, would be only $.5(.025 \times 20)$. Thus, under cued recall, $B$ words are far more likely to be guessed correctly than A words. It should be noted, however, that response omission errors did occur, thus indicating that all of the subjects did not engage in this strategy for all of the words they could not recall. Nonetheless, to the extent that this guessing strategy was used, the augmentation of recall scores for List B would be expected to be significant. whereas the augmentation of recall scores for List A would be expected to be negligible.

Two, the cues for recall of the $B$ words may have indirectly triggered serial chains formed at storage, thus facilitating recall of the B words. Evidence which suggests this explanation comes from an examination of the words comprising Lists A and B. While 11 of the 20 words of List B could be classified as both nouns and verbs or as both nouns and adjectives (star, man, light, cut, sleep, smoke, flower, fast. clean, water, and drink), only three of the words of List A could be classified as two parts of speech-noun and verb (lift, dream, and stem). It seems reasonable to expect that a list containing words which represent a larger variety of combinations of parts of speech should be more subject to serial chaining, and its associated memoryfacilitation effect, than a list consisting almost exclusively of nouns. That is, because the words of List $B$ represent a fairly heterogeneous mixture of nouns and verbs, it should be easier to relate semantically the words of List B than the words of List A. Therefore, the associative strength between adjacent words of List $B$ should be greater than the associative strength between adjacent words of List $A$. If, at recall, some of the cues did elicit their primary associates, these associates might serve to elicit adjacent words of their list even though the associates were themselves neither recallable nor recognizable. The fact that a word which is neither recallable nor recognizable can serve as the stimulus for the recall of another word is firmly established in the literature of paired associate learning. Since the probability that the cues of List B will elicit $B$ words, as primary free associates, is considerably greater than the probability that the cues of List A will elicit A words, as primary free associates, recall of $A$ words would be expected to be greater than recall of $B$ words.

In addition to these two independent explanations for the cued recall superiority of List A under silentstudy instructions, it should be noted that the words of List B were more recallable than the words of List A. Since Experiment 2 constituted a replication of Experiment 1 with but a single change (viz., the associative encoding instruction), the data of Experiments 1 and 2 of the present study were combined and analyzed in terms of a 2 by 2 by 2 between-subjects factorial design consisting of the following variables: list type (A vs. B), recall condition (cued vs. free), and encoding condition (silent study vs. associative encoding). In this way, the full power derived from performance scores of the 200 subjects $(\mathrm{df}=192)$ of Experiments 1 and 2 could be used for the determination of a highly reliable error term $(\mathrm{MSe}=10.53)$. The results of the analysis of variance for the combined data of Experiments 1 and 2 showed that more $B$ words were recalled than A words $[F(1,192)=13.4, p<.001]$, thus suggesting that, independent of the associative relationship between the $A$ and $B$ words, the words of List B were more likely to be recalled than the words of List $A$; that is, Lists $A$ and $B$ were not equal with respect to level of difficulty. This conclusion must be tempered, however, by the partial confounding caused by differences in cues associated with the words of the two lists. It may be important to note at this point that the List by Recall Condition interaction was not significant $[F(1,192)=1.00, p>.05]$, nor was the List by Encoding interaction $[F(1,192)=2.45, \quad p>05]$. Although this difference in recallability between the two lists does not, in itself, account for the superior cued recall of List $A$ under the silent-study instructions of Experiment 1, it does augment the effects that might be expected on the basis of the two explanations offered earlier.

\section{GENERAL DISCUSSION}

Consistent with the results of Humphreys and Galbraith (1975), the findings of Experiment 1 of the present study show that under silent-study encoding conditions, extralist cues are not effective in augmenting recall unless the cues bear a strong preexperimental potential for eliciting the target words as free associates. However, the findings of Experiment 2 of the present study show that, under associative encoding conditions, extralist cues are effective in augmenting recall regardless of whether the cues bear a strong preexperimental potential for eliciting the target words as free associates, or whether the target words bear a strong preexperimental potential for eliciting the cues. Although the findings of Humphreys and Galbraith are reliable, their conclusion that their results contradict the encoding specificity hypothesis does not follow because the crucial assumption underlying their procedure, the assumption that subjects instructed to study silently necessarily engage in associative encoding, appears to be untenable. The results of Experiment 2 of the present study confirmed predictions from the encoding specificity hypothesis with respect to the effectiveness of forward and backward unidirectional associates as cues in recall. Contrary to the conclusions of Humphreys and Galbraith, forward and backward unidirectional associates were equally effective as retrieval cues when subjects were instructed to engage in associative encoding at storage.

The problem of determining how subjects encode 
words to be recalled is a complex one. The findings of Dean and Ley (1977) and Ley, Dean, and Locascio (Note 1) indicate that individual differences in associative ability may be related to how subjects encode target words when instructed to study silently; the findings of Locascio and Ley (1972) and additional findings of Dean and Ley (1977) indicate that assessment characteristics of the target words (e.g., associative frequency) may be related to how subjects encode target words when instructed to study silently. The combined results of these studies suggest that encoding under silent-study instructions involves an interaction among individual difference variables, assessment characteristics of the words to be recalled, strategies of study involving serial chains or subjectively organized chains, as well as associative encoding.

\section{REFERENCE NOTE}

1. Ley, R., Dean, J., \& Locascio, D. Associative ability in free recall. Paper presented at the Psychonomic Society Meetings, St. Louis, Missouri, November 1972.

\section{REFERENCES}

Batrich, H. P. A two-phase model for prompted recall. Psychological Review, 1970, 77, 215-222.
DEAN, J., \& LEY, R. Effects of associative encoding on free recall in high and low verbal associators. Journal of Experimental Psychology: Human Learning and Memory, 1977, 3, 316-324.

Humphreys, M. S., \& Galbratth, R. C. Forward and backward associations in cued recall: Predictions from the encoding specificity principle. Journal of Experimental Psychology: Human Learming and Memory, 1975, 1, 702-710.

KEPPEL, G., \& STRand, B. Z. Free association responses to the primary purposes and other responses selected from the Palermo-Jenkins norms. In L. Postman \& G. Keppel (Eds.), Norms of word association. New York: Academic Press, 1970.

Ley, R., \& Tesiny, E. Associative reaction time, meaningfulness, and pronunciability ratings of 382 words and paralogs. Bulletin of the Psychonomic Society, 1975, 6, 645-648.

Locascio, D., \& LEY, R. Associative reaction time and meaningfulness in free recall. Joumal of Experimental Psychology, 1972, 95, 460-462.

Thomson, D. M., \& Tulving, E. Associative encoding and retrieval: Weak and strong cues. Journal of Experimental Psychology, 1970, 86, 255-262.

Tulving, E., \& Thomson, D. M. Encoding specificity and retrieval processes in episodic memory. Psychological Review, 1973, 80, 352-373.

(Received for publication February 23, 1977; revision accepted June 2,1977 .) 